\title{
Calidad del aire y su incorporación en la planeación urbana: Mexicali, Baja California, México
}

\author{
Elva Alicia Corona-Zambrano* \\ Rosa Imelda Rojas-Caldelas*
}

Resumen. A escala mundial, el problema de la contaminación del aire en los asentamientos humanos presenta una tendencia creciente, resultado del incremento en las demandas de energía y emisiones que tienen las actividades productivas y domésticas, producto de los procesos de urbanización e industrialización. Desde esta perspectiva, las ciudades no se encuentran planeadas para minimizar dichos impactos a partir de la estructura y el funcionamiento de las mismas. De ahí que el trabajo cumpla un doble propósito: por un lado, plantear la evaluación de la calidad del aire en la ciudad de Mexicali, a partir del análisis de los contaminantes: $\mathrm{O}_{3}, \mathrm{CO}, \mathrm{SO}_{2}, \mathrm{NO}_{2} \mathrm{y} \mathrm{PM} \mathrm{PM}_{10} \mathrm{y}$ su relación con la estructura urbana; y por otro, mencionar y evaluar las estrategias instrumentadas para el abatimiento de la contaminación. Finalmente, se propone un esquema de planeación y gestión urbana que incorpora aspectos de calidad del aire.

Palabras clave: Calidad del aire urbano, gestión de la calidad del aire, contaminación atmosférica, planeación urbana sustentable, planeación ambiental urbana.

Abstract. As a result of world wide urbanization and industrialization, urban air pollution shows a growing trend directly proportional to increasing demands on energy for domestic and industrial activities. From this point of view, cities along with their urban, functional and morphological structure are not being planned with pollution prevention or minimum environmental impacts in mind. This work has two purposes: first, it sets an assessment of air quality in Mexicali by analyzing $\mathrm{O}_{3}, \mathrm{CO}, \mathrm{SO}_{2}, \mathrm{NO}_{2}$ and $\mathrm{PM}_{10}$ emissions in relation to urban structure. And second, the assessment of already implemented urban strategies aimed to minimize environmental impacts. This work also presents a scheme that incorporates air quality issues into town planning and urban management.

Keywords: Urban air quality, air quality management, air pollution, sustainable urban planning, urban environmental planning.

Artículo recibido el 4 de abril de 2008 Segunda versión recibida el 11 de noviembre de 2008

Artículo aprobado el 27 de enero de 2009

\footnotetext{
* Facultad de Arquitectura y Diseño, Universidad Autónoma de Baja California. Correos electrónicos: u_eac@uabc.mx y rosa_rojas@uabc.mx
} 


\section{Introducción}

Actualmente el problema de la contaminación ambiental es un tema que se ha introducido en todos los ámbitos y en todos los niveles. Y lo que antes sólo era la preocupación de unos cuantos ambientalistas como Carson (1962), Meadows et al. (1972) y Commoner (1975), hoy es uno de los objetivos principales de la planeación y gestión del desarrollo en las diferentes regiones del planeta que pretende evaluar, prevenir y en su caso minimizar los daños al ambiente. En este sentido, dada la complejidad de la problemática ambiental asociada a las ciudades, resulta imprescindible que temas como la contaminación atmosférica sean considerados en la agenda política de los gobiernos locales tanto de países industrializados como en vías de desarrollo (Satterthwaite, 2001).

De acuerdo con Naciones Unidas (UNEP, 2006), actualmente casi la mitad de la población mundial vive en áreas urbanas y se espera que a futuro se incremente el porcentaje de población con perfil urbano. Ello va a incrementar la demanda de energía para iluminación, transporte, procesos industriales y diferentes usos domésticos que afectarán la calidad del aire por emisiones a la atmósfera de dichas actividades. En el caso particular de la contaminación atmosférica, para muchas ciudades del mundo como Hong Kong, Delhi, Bangkok, Sao Paulo, Seúl y México, ésta representa un serio problema para la salud de la población, deterioro de sus ecosistemas, infraestructura y edificaciones (McGranahan y Murray, 2003).

Las afectaciones a la salud están relacionadas con diferentes niveles de exposición a la contaminación que van desde enfermedades cardiopulmonares y respiratorias, como el asma y alergias, hasta cáncer pulmonar, donde los grupos de mayor riesgo son principalmente la población infantil y de adultos mayores (OPS, 2000; Dockery, 2001; Sorensen et al., 2003). Naciones Unidas también señala que en muchas regiones del mundo se presentan anualmente 800 mil muertes prematuras atribuidas a la contaminación del aire en áreas urbanas (UNEP, 2006). Los principales agentes ambientales implicados son los óxidos de nitrógeno (NOx) y de azufre (SOx), partículas suspendidas totales (PST), ozono $\left(\mathrm{O}_{3}\right)$, metales, compuestos orgánicos volátiles (COV) e hidrocarburos (Vargas, 2005; Seoánez, 2002). 
En América Latina, donde más de 70\% de la población vive en áreas urbanas, son muchas las ciudades que también enfrentan problemas de calidad del aire, ya que se exceden sus normas nacionales de calidad con las respectivas consecuencias, entre las que se destacan 35 mil muertes anuales (OPS-OMS, 2002); uno de los principales agentes responsables de ello son las partículas equivalentes o menores a 10 microgramos $(\mu \mathrm{m})$ en su diámetro aerodinámico, mejor conocidas como $\mathrm{PM}_{10}$. Particularmente en México, ciudades como el Distrito Federal, Guadalajara, Toluca y las fronterizas como Mexicali, también han realizado esfuerzos para atender la contaminación atmosférica (Molina y Molina, 2002; Reyna y Arriola, 2006; Osornio et al., 2007; Quintero et al., 2006). Sin embargo, en el caso particular de la ciudad de Mexicali, aún persisten los problemas de $\mathrm{PM}_{10}$, cuyos niveles de contaminación del aire han sobrepasado las normas permitidas, como en otras ciudades de América Latina (cuadro 1).

Cuadro 1. Concentración anual promedio de $\mathrm{PM}_{10}$ de algunas ciudades de América Latina y el Caribe, 2000-2004

\begin{tabular}{lcrrrrr}
\hline Ciudad & $\begin{array}{c}\text { Norma } \\
\text { anual } \\
\left(\mu \mathrm{g} / \mathrm{m}^{3}\right)\end{array}$ & \multicolumn{6}{c}{ Concentraciones anuales de $\mathrm{PM}_{10}\left(\mu \mathrm{g} / \mathrm{m}^{3}\right)$} \\
\hline Arequipa & 50 & 111 & 91 & 102 & 100 & 90 \\
Bogotá & 65 & 58 & 64 & 66 & 66 & 66 \\
Río de Janeiro & 50 & - & 39 & 40 & 53 & - \\
San Salvador & 50 & - & 60 & - & - & - \\
Cochabamba & - & - & - & 98 & 104 & 64 \\
Santiago & - & 77 & 72 & 70 & 74 & 68 \\
Sao Paulo & 50 & 52 & 49 & 51 & 48 & 41 \\
Medellín & 65 & - & - & 87 & 93 & - \\
Cd. de México & 50 & 71 & 60 & 65 & 64 & 54 \\
Mexicali $\left(^{*}\right)$ & 50 & 119.74 & 100.39 & 119.31 & 119.46 & 108.69 \\
\hline
\end{tabular}

(*) AQS-UABC, 2006.

Fuente: Adaptado de OPS-OMS, "Crecimiento urbano, contaminación atmosférica y riesgos a la salud", presentado en el Foro de Monitoreo Atmosférico y Gestión Ambiental del Aire, México, Distrito Federal, abril del 2006. 
Dada la importancia del estado y el manejo de la calidad del aire para el funcionamiento y desarrollo de los asentamientos humanos, las ciudades enfrentan retos importantes para el logro de la sustentabilidad en términos de planeación y gestión del territorio. Por ejemplo, la incorporación de la visión de la sustentabilidad se ha limitado al ordenamiento del suelo a partir del análisis de aptitud para la localización de actividades productivas, aunadas a la definición de políticas de aprovechamiento y conservación de los recursos naturales; o como en el caso del problema de la contaminación del aire, que demanda de la acción conjunta y coordinada de las diferentes instituciones y niveles de gobierno (Semarnat, 2006). Sin embargo, los temas implícitos en este problema se han tratado tradicionalmente de manera sectorial, tal es el caso de la planeación de la energía, transporte y vialidad, salud, monitoreo del aire, desarrollo urbano y medio ambiente, de tal forma que bajo este contexto pareciera que la contaminación del aire tiene que ver muy poco con la planeación urbana.

Con base en lo anterior, el presente trabajo tiene un doble propósito: por un lado, plantear la problemática de la calidad del aire en la ciudad de Mexicali a partir de un indicador de la calidad del aire, representado por los días en que fue rebasada la norma ambiental de los contaminantes objeto de este estudio y su relación con los usos del suelo dentro de la ciudad, además de mostrar las estrategias instrumentadas para abatir la contaminación a partir de la perspectiva ambiental de las instancias oficiales. Por otro lado, formular un esquema de planeación que integre diferentes estrategias sectoriales y en el que se incorporen criterios ambientales en la planeación urbana, bajo un propósito común que es el mejoramiento de la calidad del aire para, con ello, mejorar el nivel de vida de la población.

\section{Metodología}

El diagnóstico sobre la calidad del aire se hizo a partir del análisis de los niveles de concentración de los contaminantes criterio registrados por el sistema de monitoreo local.

El acceso a dicha información fue a través de la base de datos del Sistema de Calidad del Aire (AQS) 1997-2005 (AQS-UABC, 2006), de la 
Agencia de Protección Ambiental (EPA por sus siglas en inglés) de Estados Unidos de América, que para este caso y para simplificar el manejo de información, se consideró emplear la medición de la calidad del aire a través del número de días en que se violó la norma respectiva. Esta base de datos se interpoló con la utilización del programa SURFER, V.8. En relación con las características del entorno inmediato que circunda a las estaciones, se realizó un análisis de los usos del suelo dentro de un radio de influencia de 1500 metros, utilizando el sistema de información geográfica Map Info vr. 7.8; y para el caso de las estrategias instrumentadas se revisaron los documentos base y se realizaron entrevistas y cuestionarios específicos a las autoridades de los tres niveles de gobierno encargadas de los programas instrumentados localmente, esto con el fin de conocer cuáles han sido las acciones y los resultados obtenidos de las mismas durante el periodo 2000-2005.

\section{Calidad del aire en Mexicali}

Localización geográfica y condiciones ambientales

La ciudad de Mexicali se localiza en la frontera con Estados Unidos a los $32^{\circ} 39^{\prime}$ de latitud norte y $115^{\circ} 28^{\prime}$ de longitud oeste, y cuenta con una extensión de 20633.52 has (XVIII Ayuntamiento de Mexicali-IMIP, 2006). Limita al norte con Estados Unidos de América y en la periferia con áreas agrícolas (figura 1).

Figura 1. Ubicación de Mexicali

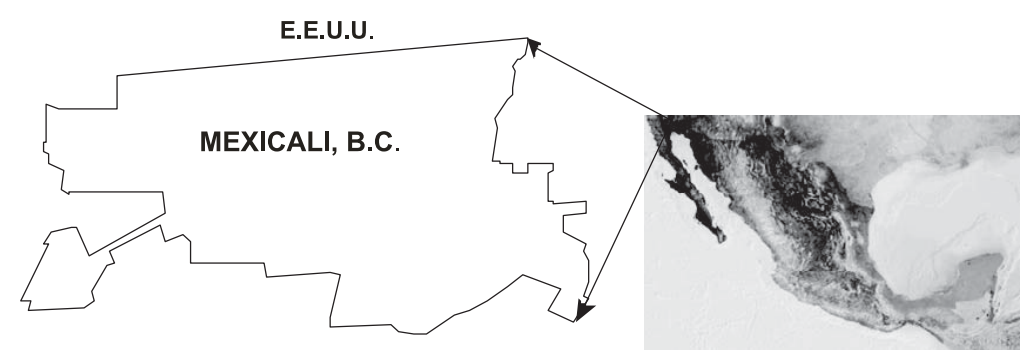


En general, la ciudad se ubica en una zona árida denominada Bajo Delta del Río Colorado, perteneciente a la región fisiográfica del desierto sonorense, región caracterizada en temporadas por el acarreo de polvo y arena provenientes del desierto tanto de la parte mexicana como de la estadounidense. Asimismo, el Bajo Delta ha sido el sustento de áreas agrícolas en ambos lados de la frontera, con las implicaciones que ello tiene principalmente en contaminación del aire, resultado de las quemas agrícolas y la aplicación aérea de plaguicidas.

Entre sus principales características ambientales, el clima corresponde al tipo BW, particularmente el BW( $\left.\mathrm{h}^{\prime}\right) \mathrm{hs}\left(\mathrm{x}^{\prime \prime}\right)(\mathrm{e})$, lo que significa un clima muy árido, seco, cálido, con lluvias en invierno y muy extremoso (García, 1988); con temperaturas extremas que en verano alcanzan los $54^{\circ} \mathrm{C}$ y en el invierno llegan a descender hasta $-7^{\circ} \mathrm{C}$; la temperatura media anual es de $22.5^{\circ} \mathrm{C}$ (Venegas, 2000). La precipitación promedio anual es de $75 \mathrm{~mm}$ (García et al., 2002). Además, las condiciones climáticas de la región están determinadas por la presencia del sistema anticiclónico subtropical semipermanente del Pacífico, a lo que se atribuye que los alrededores de la costa de California y los valles de Imperial y Mexicali generalmente experimenten cielos claros, muy baja humedad y veranos extremadamente calurosos, inviernos templados y poca precipitación, así como vientos dominantes que provienen del oeste-noroeste en invierno y primavera y suroeste-sureste en el verano.

$\mathrm{Al}$ igual que otras zonas fronterizas, Mexicali no sólo presenta similitud con la ciudad vecina de Calexico, California, en cuanto a las características climáticas, topográficas y la dinámica socioeconómica y cultural, sino que también el flujo bidireccional se presenta en lo relativo a la contaminación atmosférica.

Es importante destacar que el origen y desarrollo de la ciudad se encuentra en la actividad agrícola de la región. Actualmente la agricultura tiene el segundo lugar de ocupación dentro de la población económicamente activa, encontrándose entre los principales cultivos el trigo, las hortalizas y el algodón (XVIII Ayuntamiento de MexicaliIMIP, 2006).

Por otro lado, a escala urbana, Mexicali presenta un nivel de motorización de un vehículo por cada tres habitantes, mientras que el 
transporte público mueve solamente alrededor de la tercera parte de la población (INEGI, 2004). Asimismo, la industria maquiladora ha sido y es uno de los motores de la economía local. Estas actividades han contribuido en el tiempo al agravamiento de la calidad del aire, asunto que por el impacto que ha tenido a escala local ha sido considerado tema de interés binacional.

Estaciones de monitoreo y usos del suelo

La ciudad de Mexicali cuenta con un sistema de monitoreo atmosférico que se conforma por seis estaciones; en la figura 2 se muestra su ubicación, en tanto que el cuadro 2 describe la relación entre las mismas y los usos de suelo circundantes. Los contaminantes monitoreados básicamente son ozono $\left(\mathrm{O}_{3}\right)$, monóxido de carbono $(\mathrm{CO})$, dióxido de azufre $\left(\mathrm{SO}_{2}\right)$, dióxido de nitrógeno $\left(\mathrm{NO}_{2}\right)$ y material particulado $\left(\mathrm{PM}_{10}\right)$, esto derivado de un acuerdo entre los gobiernos de Estados Unidos

Figura 2. Usos de suelo en Mexicali

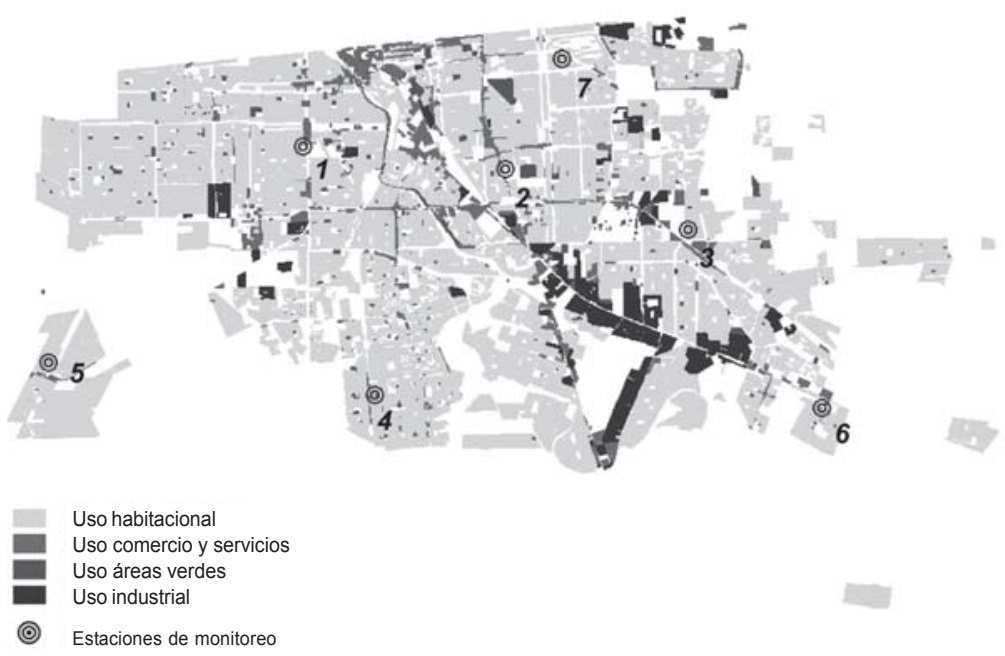

Fuente: Elaboración propia a partir de la información del XVIII Ayuntamiento de Mexicali-IMIP, 2006. 


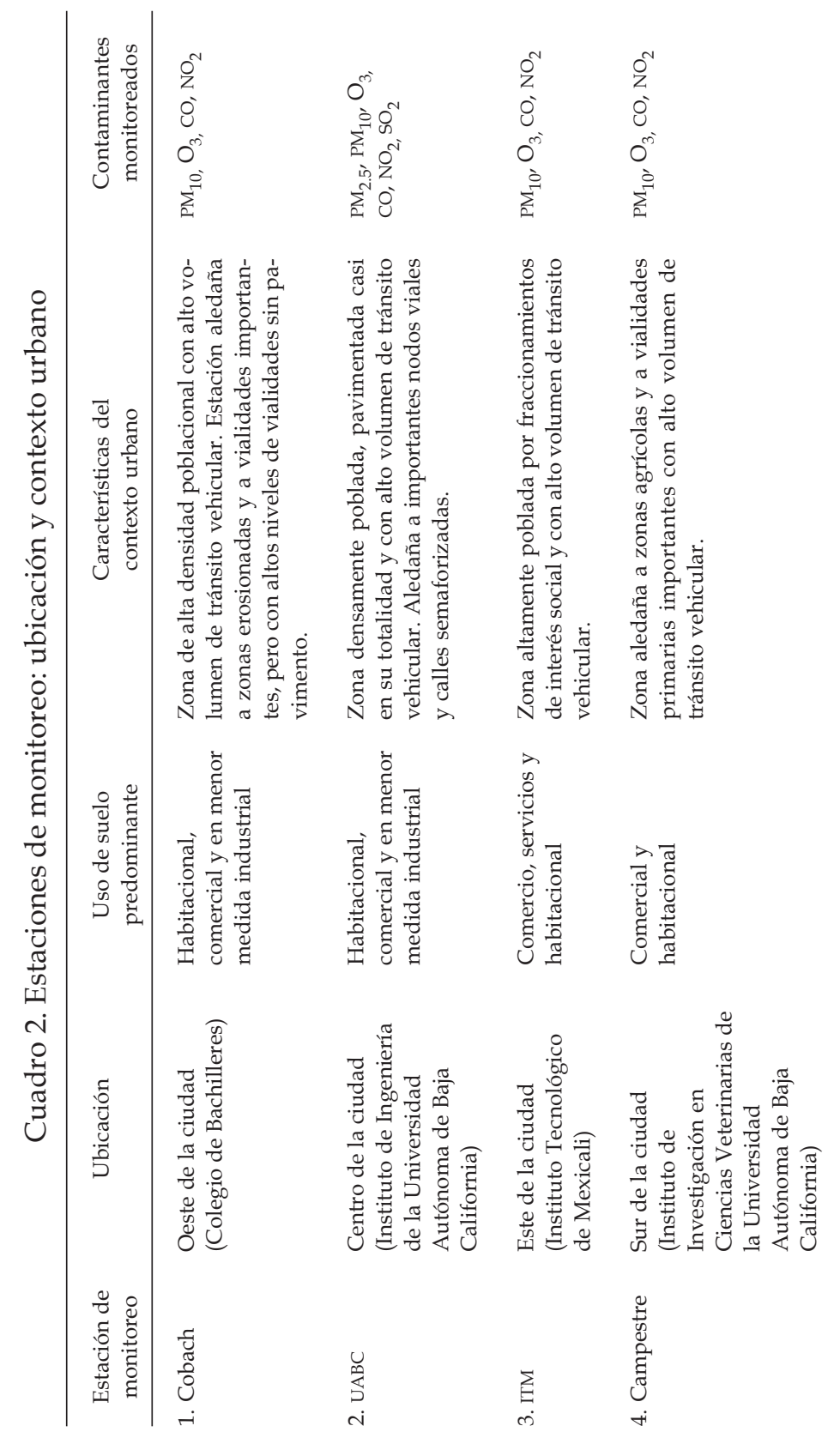




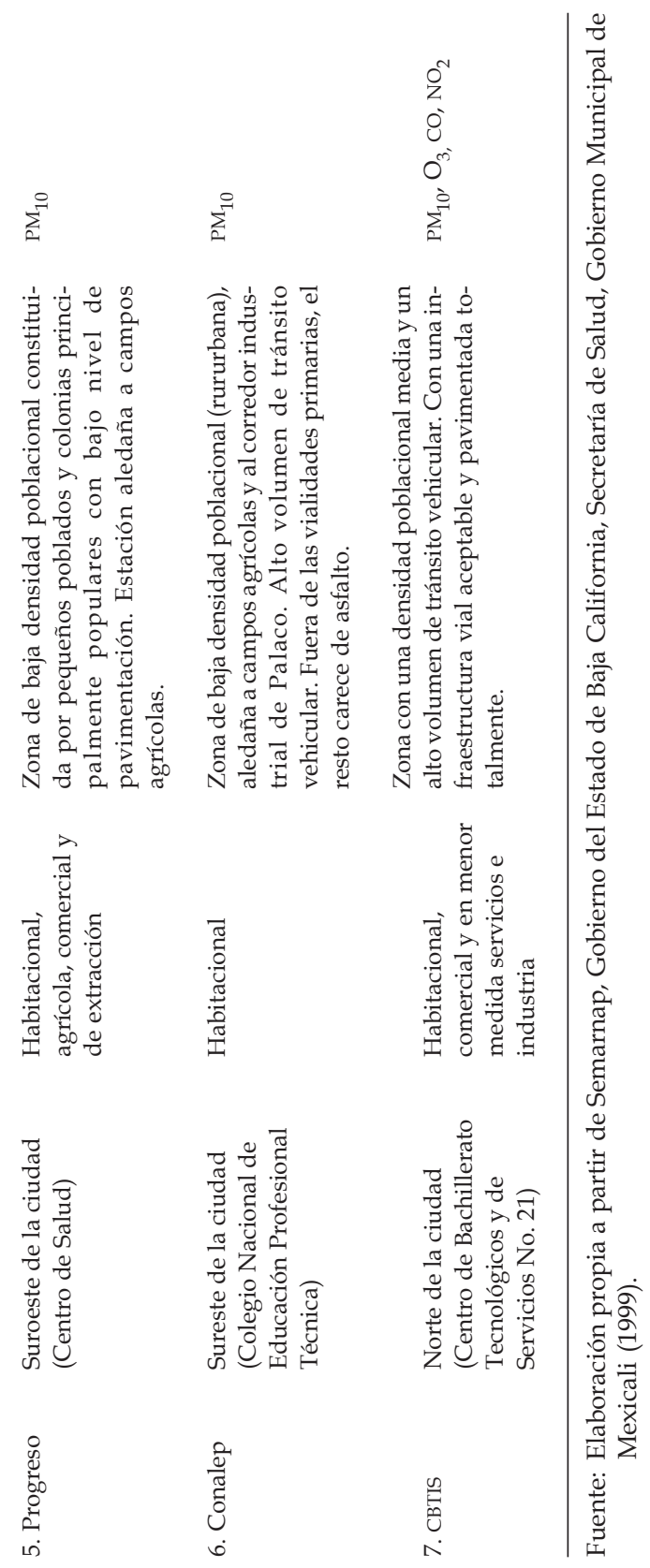


de América y México a través del programa Binacional Frontera XXI, que posteriormente se convirtió en Programa Frontera 2012. En el caso de las estaciones Progreso y Conalep, solamente miden $\mathrm{PM}_{10}$. Es conveniente anotar que si bien la estación CBTIS deja de operar a partir del 2002 para ser reubicada al sur de la ciudad en el 2005, bajo el nombre de Campestre, su inclusión en el estudio es importante ya que arroja información valiosa de la calidad de aire en su área de influencia entre 1997 y 2001.

\section{Principales contaminantes atmosféricos monitoreados en Mexicali}

Los resultados obtenidos de los monitoreos realizados entre 1997 y 2005 en la ciudad de Mexicali reflejan la dinámica que tiene la ciudad a partir de las actividades económicas que le dan sustento y de las condiciones ambientales relacionadas con su localización geográfica.

Las principales causas de la contaminación del aire se encuentran en la generación de energía, vehículos automotores, industria, rellenos sanitarios a cielo abierto, tormentas de polvo características de la región, el tránsito de vehículos sobre vialidades no pavimentadas, así como las quemas agrícolas en ambos lados de la frontera (Mendoza et al., 2004).

Los datos actuales generados por el sistema de monitoreo coinciden con los reportes anteriormente realizados en 1997 y 1998 por la Semarnap, el gobierno del estado de Baja California, la Secretaría de Salud y el gobierno municipal de Mexicali (1999), que destacaban como principal problema las partículas $\mathrm{PM}_{10}, \mathrm{CO}_{\text {y }} \mathrm{O}_{3}$, contaminantes que se concentraban principalmente en las estaciones Cobach, Progreso, CBTIS y UABC. Sin embargo, los datos de 2005 (AQS-UABC, 2006) reportan una reducción sensible en los niveles de contaminación, aun cuando actualmente los niveles de concentración de los mismos rebasan los niveles permisibles para $\mathrm{PM}_{10} \mathrm{y}_{3}$.

En el caso de $\mathrm{PM}_{10}$ para el periodo 1997-2005, ninguna estación ha estado por debajo de la norma; sin embargo, las estaciones ITM, СBTIS y Campestre presentan los registros más bajos del periodo analizado, mientras que las estaciones Progreso y Cobach, seguidas de la UABC, 
presentan el mayor número de violaciones a la norma, principalmente la estación Progreso que registró 49, 40 y 46 días en 2000, 2001 y 2004 respectivamente. Por su parte, la estación Cobach registra el mayor número de violaciones durante los años 1999 y 2000, con 26 días. La estación Conalep presenta un mayor número de violaciones durante 1999, disminuyendo éstas entre 2001 y 2005 (cuadro 3).

El co para el periodo de análisis, con excepción de la estación Campestre y parcialmente Cobach, muestra en la mayoría de las estaciones una tendencia ascendente entre 1997 y 1999, la cual se revierte del 2000 al 2005, al reducirse el número de violaciones también en la mayoría de las estaciones, pues cabe recordar que la estación CBTIS cierra operaciones para el 2002. Las estaciones Cobach y UABC, seguidas del ITM, presentan el mayor número de violaciones a la norma, principalmente la estación Cobach que registró en el año 1998 un total de 68 violaciones. Para el 2005 ya no se registra violación a dicha norma (cuadro 4).

$\mathrm{El}_{3}$ manifiesta una tendencia general, si bien a la baja, en la mayor parte de las estaciones, respecto a los niveles registrados durante 1997; para los años 1999 y 2001 se registra nuevamente un repunte en estaciones como UABC y Cobach respectivamente. Esta última durante 2002 logró reducir el número de violaciones a un día, hasta que en

Cuadro 3. Número de días en que se rebasó la norma para $\mathrm{PM}_{10}$, 1997-2005

\begin{tabular}{lcrrrccc}
\hline Año & ITM & UABC & Cobach & CBTIS & Campestre & Conalep & Progreso \\
\hline 1997 & 0 & 5 & 8 & 0 & - & 4 & 21 \\
1998 & 0 & 3 & 11 & 1 & - & 2 & 23 \\
1999 & 1 & 5 & 26 & 2 & - & 5 & 30 \\
2000 & 1 & 8 & 26 & 0 & - & 1 & 49 \\
2001 & 1 & 1 & 13 & 1 & - & 4 & 40 \\
2002 & 3 & 2 & 17 & - & - & 3 & 34 \\
2003 & 0 & 2 & 18 & - & - & 1 & 35 \\
2004 & 0 & 3 & 14 & - & 0 & 0 & 46 \\
2005 & 0 & 1 & 10 & - & 1 & 0 & 38 \\
\hline
\end{tabular}

Fuente: AQS-UABC, 2006. 
Cuadro 4. Número de días en que se rebasó la norma para CO, 1997-2005

\begin{tabular}{rrrccc}
\hline Año & ITM & UABC & Cobach & CBTIS & Campestre \\
\hline 1997 & 10 & 27 & 41 & 38 & - \\
1998 & 17 & 42 & 68 & 55 & - \\
1999 & 18 & 49 & 56 & 56 & - \\
2000 & 15 & 39 & 37 & 41 & - \\
2001 & 1 & 22 & 46 & 26 & - \\
2002 & 5 & 29 & 42 & 14 & - \\
2003 & 3 & 13 & 28 & - & - \\
2004 & 3 & 4 & 18 & - & - \\
2005 & 0 & 0 & 0 & - & 0 \\
\hline
\end{tabular}

Fuente: AQS-UABC, 2006.

2005 ya no registró violación a la norma, contrariamente al patrón registrado por la UABC, donde del 2002 al 2004 se presenta una reducción paulatina al número de violaciones, y nuevamente en el 2005 sufre otro repunte, con siete días en los que los niveles de concentración de dicho contaminante exceden los límites permitidos (cuadro 5).

Cuadro 5. Número de días en que se rebasó la norma para $\mathrm{O}_{3}$, 1997-2005

\begin{tabular}{lrrrcc}
\hline Año & ITM & UABC & Cobach & CBTIS & Campestre \\
\hline 1997 & 16 & 16 & 12 & 8 & - \\
1998 & 14 & 6 & 5 & 5 & - \\
1999 & 3 & 11 & 12 & 13 & - \\
2000 & 2 & 5 & 6 & 4 & - \\
2001 & 7 & 10 & 11 & 1 & - \\
2002 & 2 & 7 & 1 & - & - \\
2003 & 6 & 6 & 1 & - & - \\
2004 & 3 & 4 & 1 & - & - \\
2005 & 1 & 7 & 0 & - & 0 \\
\hline
\end{tabular}

Fuente: AQS-UABC, 2006. 
Estrategias para mejorar la calidad del aire en Mexicali:

la perspectiva de la Semarnat

En respuesta a los problemas de contaminación atmosférica en la ciudad de Mexicali, diferentes instancias y niveles de gobierno instrumentaron programas y acciones como las formuladas a nivel federal en el Programa para el Mejoramiento de la Calidad del Aire en Mexicali 2000-2005 (Semarnap, Gobierno del Estado de Baja California, Secretaría de Salud, Gobierno Municipal de Mexicali, 1999), mejor conocido como "Proaire", estrategias que buscaban reducir las emisiones de los diferentes contaminantes que rebasaban la normatividad correspondiente.

Las estrategias estuvieron dirigidas hacia vehículos, gestión urbana y transporte; recuperación ecológica e investigación; y acuerdos internacionales, y de ellas se desprendieron 27 acciones. Para 2006 se tenía conocimiento solamente (Gordillo, 2006; IIS-JUEBC, 2006; Páez, 2007) de la operación de seis acciones específicas:

- Transferencia, operación y financiamiento de la red de monitoreo atmosférico por parte de Estados Unidos hacia la parte mexicana; red que permite consultar casi en tiempo real las condiciones atmosféricas de la ciudad.

- El Programa Integral de Pavimentación y Calidad del Aire (PIPCA), que ha operado durante 2003 y 2004, mejorando el índice de urbanización con las obras de pavimentación que beneficiaron a alrededor de 20 colonias de la ciudad, con una superficie total de 214 has (IIS-JUEBC, 2006).

- Estudio integral del transporte público y su mejoramiento, mismo que propone el reemplazo de una parte de la flotilla por modernos y equipados autobuses; y la instrumentación de un sistema automatizado de semaforización en los principales nodos viales de la ciudad.

- Cultura ambiental y forestación urbana, programa operado en escuelas primarias y áreas recreativas, dirigido también a la población en general con el objeto de contribuir a que la comuni- 
dad participe y comprenda la dimensión de la problemática ambiental.

- Vehículos. En este tema se trabaja en la implantación del Programa de Verificación Vehicular, orientado al control de emisiones que genera el transporte público y privado, proyecto realizado en el 2006 y que actualmente se encuentra sujeto de aprobación por parte del cabildo del municipio de Mexicali.

- Puentes y pasos a desnivel. Aquí cabe destacar que, aun cuando el Proaire no consideraba estrategias y acciones viales, el programa de Puentes y Pasos a Desnivel, junto con el de Semaforización, han contribuido a reducir el efecto de los puntos "calientes" o nodos de congestionamiento vial sobre vialidades primarias en términos de emisiones a la atmósfera por el lento tráfico.

\section{Planeación urbana y calidad del aire}

La contaminación atmosférica en el contexto de la planeación urbana

En la actualidad, en la ciudad de Mexicali los efectos y las consecuencias de los daños ocasionados por la baja calidad del aire son difíciles de precisar, ya que si bien por un lado es evidente la violación de la norma ambiental de contaminantes como el $\mathrm{PM}_{10}$, $\mathrm{CO}$ y $\mathrm{O}_{3}$, por el otro urbanísticamente se ha consecuentado la extensión desmedida de la mancha urbana y otros aspectos que de igual manera han frenado el avance en materia de planeación y gestión del desarrollo urbano ambiental local.

En particular, en el tema de la calidad del aire, aun cuando éste es responsabilidad de los tres niveles de gobierno, no existe o es escasa la coordinación entre las diferentes instancias de acuerdo a sus prioridades, objetivos y programas de trabajo. En Mexicali la calidad del aire ha cobrado relevancia más por ser un problema binacional que por un interés local, a pesar de que la ley establece claramente que son la Semarnat y el Instituto Nacional de Ecología (INE) los responsables de su atención. 
A partir de los resultados obtenidos se encuentra que el $\mathrm{O}_{3}$ aún se presenta en las estaciones UABC, Cobach e ITM; estas dos últimas, aunque en menor proporción, continuaron rebasando la norma durante 2005. Estas estaciones representan a zonas predominantemente urbanas, con densidades de población de moderadas a altas, con núcleos de concentración de equipamiento urbano de importancia urbana y regional o bien de actividades industriales, lo que genera grandes volúmenes de tráfico vehicular por la dinámica de traslados de población y bienes dentro de la ciudad.

Asimismo, el $\mathrm{PM}_{10}$ continúa presentándose principalmente en estaciones como Progreso y Cobach, y en menor medida en UABC; esto se explica en los dos primeros casos por ser zonas periféricas de la ciudad con bajos niveles de urbanización y aledañas a áreas agrícolas, las cuales se ven afectadas por la presencia de polvos producto del paso de vehículos por vialidades sin pavimento, tierras agrícolas productivas que practican las quemas periódicamente, o bien porque son tierras carentes de cobertura vegetal y por tanto sujetas a erosión por viento. El caso de la estación $\mathrm{UABC}$ representa una excepción por estar en una zona con buena urbanización y menor porcentaje de vialidades sin pavimentar, caso que habría que estudiar a otro nivel de detalle.

Es importante anotar que en el caso de la industria, aun cuando los niveles tanto de $\mathrm{NO}_{2}$ y $\mathrm{SO}_{2}$ no han presentado violaciones a la norma desde el 2003, dentro de la perspectiva de la prevención de la contaminación ambiental, es menester considerar la importancia de este sector en el desarrollo económico de la localidad a futuro, ya que de mantenerse la tendencia al aumento en la cantidad y tipo de industrias que pretenden promoverse para el proyecto industrial de impacto binacional Silicon Border, seguramente cambiarían las tendencias de contaminación tanto por $\mathrm{NO}_{2}$ como por $\mathrm{SO}_{2}$.

Con base en lo anterior, se identifica que Mexicali tiene problemas de una deficiente planeación o escasa información relacionada con los siguientes temas:

- Número creciente de vehículos en circulación, la mayoría de ellos importados de Estados Unidos y una con antigüedad de por lo menos cinco años. 
- Transporte público que moviliza sólo a una tercera parte de la población.

- La ausencia de un inventario exhaustivo de contaminantes totales generados por el sector industrial, de ahí que solamente se ha procurado estimar la importancia de las diferentes industrias a partir de métodos indirectos (Semarnap, 1997).

- Industrias contaminantes instaladas en la ciudad, tales como industria eléctrica, metalúrgica, química, papelera y de alimentos, por mencionar algunas.

- Desarrollo agrícola que emplea diversos tipos de agroquímicos para incrementar la productividad y de plaguicidas para el control de enfermedades, mismos que por el comportamiento del viento distribuyen la contaminación.

- Carencia de normatividad local y capacidad de las instituciones para tener un control de los bancos de materiales circundantes a la ciudad.

- Usos de suelo incompatibles, desarrollos habitacionales, industriales, sitios de disposición final de residuos sólidos y residuos no peligrosos.

- Terrenos agrícolas abandonados y grandes lotes baldíos que a falta de cobertura vegetal se erosionan fácilmente.

- Déficit de $49.9 \%$ de áreas verdes en la ciudad (XVIII Ayuntamiento de Mexicali-IMIP, 2006), además de la presión a que están sujetas las áreas de conservación y de alta productividad agrícola por los desarrolladores inmobiliarios.

Integrando la planeación y gestión urbana con la calidad del aire

La planeación urbana enfrenta diferentes desafíos, como por ejemplo lograr la comprensión de su razón de ser, así como de la dimensión que tiene la creciente dinámica urbana y el deterioro ambiental que caracteriza a los actuales centros urbanos (Ridell, 2004). También se encuentra ante la disyuntiva de redefinirse y contar con políticas acordes a la compleja realidad, o bien convertirse en un instrumento más que sólo mitigue las eventualidades urbano-ambientales de los asentamientos humanos. En este sentido, para Layard y Davoudi (2001) 
es la planeación la que puede contribuir a determinar las prioridades y por ende las acciones requeridas dentro de una esfera espacial al considerar las preocupaciones económicas, sociales y ambientales.

La planeación urbana, incluyendo el manejo ambiental, ha sido activada en un gran número de ciudades, ya que cada vez son más las comunidades que han empezado a introducir estudios de planeación y manejo urbano-ambiental (Hallsmith, 2003). En este nivel es imprescindible retomar enfoques metodológicos interdisciplinarios que ayuden a integrar acciones conjuntas entre varios sectores para realizar una adecuada planeación de las ciudades que las oriente en el tiempo hacia el logro de la sustentabilidad (Ravinovitch y Leitman, 2004).

Dentro de la planeación y gestión del territorio a escala local, si bien la planeación de los asentamientos humanos sigue concentrándose en el manejo de los usos del suelo, es importante que se consideren algunos criterios ambientales tanto al momento de realizar los diagnósticos como en la formulación de estrategias que permitan reducir el impacto al ambiente producto de las actividades propuestas, y definitivamente, apoyarse en otros instrumentos que coadyuven a dicho propósito (Roseland, 1998; Ridell, 2004).

Lo anterior contribuirá en el manejo de temas como la calidad del aire de los centros urbanos, que en este caso requiere contar con herramientas que permitan redimensionar su problemática y hacer interactuar la información tanto de tipo ambiental como de urbanización, industrialización y transporte, con el objeto de buscar soluciones integrales y sustentables.

A continuación se presentan los diferentes aspectos de la ciudad que requieren ser considerados integralmente por la planeación urbana para atender los problemas de la contaminación atmosférica que en ella se presentan (Corona y Rojas, 2008). Básicamente se refieren a elementos que interactúan en los procesos de planeación y gestión de la calidad del aire urbano y que tienen que ver con la caracterización de las actividades productivas: industria, agricultura, actividades extractivas, normatividad y su vigilancia, y áreas verdes, entre otros (figura 3).

- Industria. Integrar la planeación del uso del suelo con la evaluación ambiental estratégica, la aplicación de la normatividad en 
Figura 3. Esquema de planeación urbana y gestión de la calidad del aire

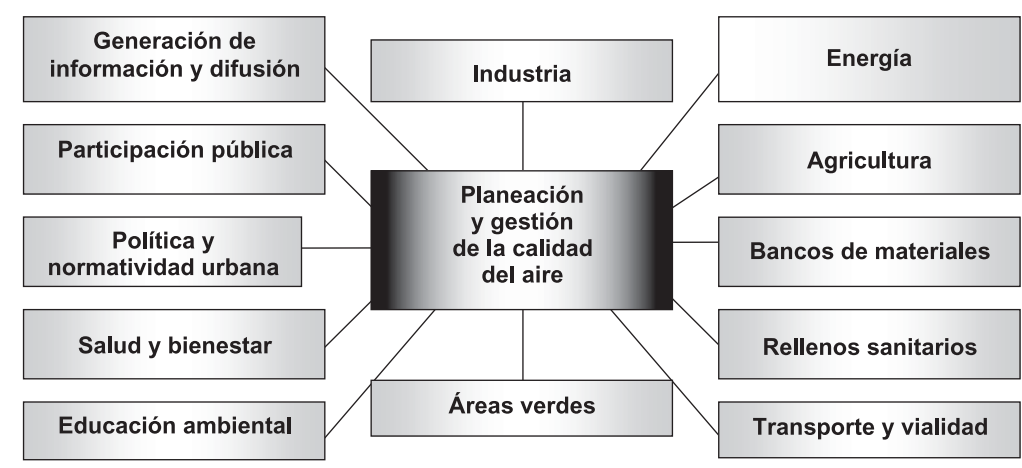

Fuente: Corona, 2008.

la autorización y el seguimiento de la operación de la industria, publicación y comunicación al público de las características de las emisiones al aire de las empresas, además de promover el ingreso de las empresas a los programas de certificación ambiental y desincentivar el asentamiento de las industrias relacionadas con la industria eléctrica, metalúrgica, química, papelera y de alimentos, para disminuir la participación de aquellas ramas de la industria identificadas como generadoras de emisiones considerables y altamente contaminadoras.

- Agricultura. Importante contribución tendrán el control y monitoreo de quemas agrícolas y de aplicación de agroquímicos y plaguicidas en áreas productivas y la creación de barreras vegetales de amortiguamiento.

- Bancos de materiales. Regulación y monitoreo de emisiones en la operación de éstos.

- Rellenos sanitarios. Determinación oportuna y adecuada de la ubicación del sitio para el confinamiento de residuos sólidos o peligrosos y monitoreo de emisiones.

- Transporte y vialidad. Promover mediante la regulación del uso del suelo un patrón compacto de ciudad de mayor densidad de población, que tienda a su vez a reducir las distancias entre es- 
pacios habitacionales y de trabajo, acompañado por un incremento en la infraestructura vial primaria y mejores coberturas de pavimentación. Incentivar e incrementar la movilización por transporte público, con unidades nuevas, adecuadas para las condiciones climáticas de la región. Además, es primordial regular el parque vehicular y la circulación de unidades con antigüedad mayor a 10 años, acreditar positivamente la verificación de emisiones y promover incentivos económicos o fiscales para la sustitución del parque vehicular de mayor antigüedad.

- Áreas verdes. Abatir el déficit de las mismas y promover su aprovechamiento para contribuir en el mejoramiento de la calidad del aire, a través del empleo de las especies adecuadas para la región.

- Energía. Promover el mejoramiento de la calidad de los combustibles para el transporte, pero también la generación y el empleo de energías alternas en el uso industrial, doméstico y comercial para disminuir el impacto de la quema de combustibles fósiles.

- Salud. Seguimiento sistematizado de las enfermedades provocadas por los altos índices de contaminación, además de un programa preventivo que informe y oriente a la población de las medidas que requiere tener en cuenta a fin de preservar su salud.

- Política y normatividad ambiental urbana. Tanto la definición de políticas como de normas acordes a la realidad imperante son elementos indispensables para definir e instrumentar las estrategias y los programas orientados a la atención de la problemática ambiental y urbana de los asentamientos humanos.

- Generación de información ambiental. Desarrollar indicadores de presión y respuesta que sean complementarios a los del estado (condiciones actuales de la calidad del aire), así como generar información a partir de los sistemas de monitoreo y los inventarios de emisiones, que incidan positivamente en el diseño de políticas públicas para medir el desempeño de los programas que generan los diferentes sectores.

- Información y difusión. Es recomendable para que las políticas, programas y acciones no sólo sean tomadas en cuenta por las autoridades en el diseño e instrumentación de estrategias y pro- 
gramas exitosos de mejoramiento de la calidad del aire, sino también para que la población sea informada adecuada y oportunamente sobre la calidad del aire y cuáles son las condiciones atmosféricas en las que se deben extremar precauciones. Estos aspectos contribuirán significativamente a fomentar la educación y cultura ambiental.

- Educación ambiental. Rubro importante para generar en la población una cultura de interés y conciencia por los temas ambientales, particularmente por el estado y las condiciones que guarda la calidad del aire local. Dada la importancia y magnitud del tema, resulta conveniente no reducir las estrategias de educación ambiental a las instrumentadas por las instancias gubernamentales. El sector educativo requiere incorporar en sus programas de estudios materias inherentes al medio ambiente y a las perspectivas que éste tiene en los tiempos modernos.

\section{Conclusiones}

En la ciudad de Mexicali, además de que no es satisfactoria la calidad del aire, el despliegue de esfuerzos sectorizados por parte de las instancias gubernamentales, aun cuando han contribuido en la declinación significativa de contaminantes como el co y en menor medida de $\mathrm{PM}_{10} \mathrm{y} \mathrm{O}_{3}$, no ha sido suficiente para mantener bajo la norma a los mismos. Destacan, por tanto: 1. la carencia de una visión integral de los problemas generados en las áreas urbanas; 2 . la limitada coordinación interinstitucional en la atención y solución de los problemas urbano-ambientales; 3 . los objetivos, metas, recursos y prioridades determinados separadamente; y 4 . la falta de información o accesibilidad a ella en los tres niveles de gobierno.

Por lo anterior, es imprescindible que la planificación urbana incorpore la dimensión ambiental en forma sistemática y continua como un instrumento para garantizar la viabilidad de la expansión y crecimiento de las ciudades.

El tema de la calidad del aire asociado a los asentamientos humanos y los aspectos inherentes al mismo, requiere ser tratado integralmente en los distintos niveles institucionales, jurídicos, nor- 
mativos y sociales, ya que su postergación puede no sólo consentir la sobreexplotación de recursos naturales, sino también la descoordinación, duplicación e imprecisión de tareas tanto en la planeación como en la toma de decisiones, así como en el seguimiento y monitoreo de las acciones instrumentadas, lo que traería como consecuencia no sólo el retraso de medidas que pueden ser ya necesarias o urgentes, sino también la agudización de problemas como el de la contaminación atmosférica, amenazando drásticamente la calidad del aire y por ende la salud y el medio ambiente en general de los asentamientos humanos.

\section{Bibliografía}

AQS-UABC (2006), Base de datos: contaminantes $\mathrm{PM}_{10^{\prime}} \mathrm{NO}_{2^{\prime}} \mathrm{CO}_{2^{\prime}} \mathrm{SO}_{2}$ y $\mathrm{O}_{3}$ Reporte Técnico de Investigación, Mexicali, BC México, UABC.

Carson, R. (1962), Silent Spring, EE.UU., A Mariner Book.

Commoner, B. (1975), Making Peace with the Planet, Nueva York, The New Press.

Corona, Z.E.A. (2008), Sistema de indicadores para la planeación y gestión sustentable de las ciudades: calidad del aire en Mexicali, B.C., tesis de doctorado en Ciencias, Instituto de Ingeniería, UABC, Mexicali, B.C.

y R. Rojas-Caldelas (2008), “Environmental Planning and Management of Air Quality: The Case of Mexicali, Baja California, Mexico", en WIT Transactions on Ecology and the Environment, vol. 116, Reino Unido, pp. 409-418.

Dockery, D.W. (2001), “Epidemiologic Evidence of Cardiovascular Effects of Particulate Air Pollution", Environmental Health Perspectives, 109 (4), pp. 483-486.

García, R.O., C. Martínez, J. Cervantes y A. Tejeda (2002), “A Comparative Simple Method for Human Bioclimatic Conditions Applied to Seasonally Hot/Warm Cities of México", Atmósfera, vol. 15, núm. 1, pp. 55-56.

García, E. (1988), Modificación al sistema de clasificación climática de Köppen, México, UNAM, Instituto de Geografía. 
ESTUDIOS FronTERIZOS, VOL. 10, NÚM. 20, JULIO-DICIEMBRE 2009

García Cuéllar, C., M.A. Reyna, M. Quintero y J. Landeros (2007), “How Much Soil Content is Involved in Urban 10PM -Induced Toxicity?", Memories of the Us-Mexico Binational Center for Environmental Science and Toxicology, Tucson, Arizona.

Hallsmith, G. (2003), The Key to Sustainable Cities: Meeting Human Needs Transforming Community Systems, Canadá, New Society Publishers.

INEGI (2004), "Índice de motorización por entidad federativa, 1980 a 2004", en Estadísticas ambientales: transporte, en línea: http:// www.inegi.gob.mx/est/contenidos/espanol/rutinas/ ept.asp?t=mamb137\&c=5885, recuperado el 15 de febrero de 2007.

IIS-JUEBC (2006), Evaluación de impacto del programa integral para el mejoramiento de la calidad de vida del estado de Baja California, Reporte técnico de investigación, UABC, Mexicali, BC.

Layard, A. y S. Davoudi (2001), "Sustainable Development and Planning: An Overview“, en A. Layard, S. Davoudi y S. Batty (eds.), Planning for a Sustainable Future, Estados Unidos y Canadá, Spon Press.

McGranahan, G. y F. Murray (2003), Air Pollution and Health in Rapidely Developing Countries, Londres, Earth Scan.

Mendoza, A., M. García y E. Pardo (2004), “Air Quality Information Catalogue for the Mexicali-Imperial Valley Border Region: Mexicali-Imperial Valley Air Quality Modeling and Monitoring Program in Support of LASPAU's Border Ozone Reduction and Air Quality Improvement Program“, Draft Final Report LASPAUTecnológico de Monterrey, México, ITM.

Meadows, D., J. Randers y W. Behrens (1972), Los límites del crecimiento, México, Fondo de Cultura Económica.

Mitchell, G. (2004), "Forecasting Urban Futures: A Systems Analytical Perspective on the Development of Sustainable Urban Regions", en M. Purvis y A. Grainger (eds.), Exploring Sustainable Development: Geographical Perspectives, Reino Unido y Estados Unidos, Earthscan.

Molina, L. y M. Molina (2002), Air Quality in the Mexico Megacity: An Integrated Assessment, Boston y Londres, Kluwer Academic Publishers. 
OPS (2000), La salud y el ambiente en el desarrollo sostenible, núm. 572, Washington, D.C., OPS.

OPS-OMS (2002), La salud en las Américas, núm. 587, vol. I, Washington, D.C., OPS.

Osornio, A.R., G. Flores, I. Vázquez, J. Miranda, L. Sevilla, I. Rosas, L. Rojas-Bracho, I. Clark, C. García Cuellar, M.A. Reyna, M. Quintero y J. Landeros (2007), "How much soil content is involved in urban PM10-induced toxicity?"; Memories of the U.S.-Mexico Binational Center for Environmental Science and Toxicology, Tucson, Arizona.

Quintero, M., M.A. Reyna, K. Collins, S. Guzmán, B. Powers y A. Mendoza (2006), "Issues Related to Air Quality and Health in the California-Baja California Border Region", en R. Pumfrey, The Us-Mexican Border Environment: Binational Air Quality Management, SCERP Monograph Series núm. 14 San Diego, San Diego State University Press.

Reyna M.A. y H.G. Arriola (2006), “El estudio de las principales enfermedades respiratorias y los contaminantes del aire en Mexicali, Baja California", en Contaminación y medio ambiente en Baja California, Miguel Ángel Porrúa-UABC.

Riddell, R. (2004), Sustainable Urban Planning, Reino Unido, Blackwell Publishing.

Rabinovitch, J. y J. Leitmann (2004), “Urban Planning in Curitiba", en S. Wheeler y T. Beatley (eds.), The Sustainable Urban Development Reader, Estados Unidos y Canadá, Routledge.

Roseland, M. (1998), Toward Sustainable Communities: Resources for Citizens and their Governments, Canadá, New Society Publishers.

Satterthwaite, D. (2001), The Earthscan Reader in Sustainable Cities, Londres, Earthscan.

Semarnap, Gobierno del Estado de Baja California, Secretaría de Salud, Gobierno Municipal de Mexicali (1999), Programa para mejorar la calidad del aire de Mexicali 2000-2005, México, Semarnap.

Semarnat (2006), La gestión ambiental en México, México, Semarnat. (1997), Sistema integrado de regulación y gestión ambiental de la industria en México, México, Semarnat. 
ESTUdiOS FronTERIZOS, VOL. 10, NÚM. 20, JULIO-DICIEMBRE 2009

Seoánez, M. (2002), Tratado de contaminación atmosférica: problemas, tratamiento y gestión, España, Mundi-Prensa.

Sorensen, M., M. Daneshvar, B. Hansen, M. Dragsted, L. Hertel, L. Knudsen y S. Loft (2003), "Personal PM2.5 Exposure and Markers of Oxidative Stress in Blood", Environmental Health Perspectives, 111.2, pp. 161-166.

Venegas, R. (2000), “El uso de la flora urbana en ciudades con clima árido seco extremoso", en Ciudad, salud y medio ambiente, Red Nacional de Investigación Urbana, Universidad Autónoma de Puebla, México, pp. 106-116.

UNEP (2006), "Urban Outdoor Pollution", en Global Environmental Outlook Year Book 2006, United Nations Environmental Programme, pp. 47-58. http://www.unep.org/geo/yearbook/ yb2006/057.asp, recuperado el 9 de enero de 2007.

Vargas, F. (2005), "La contaminación ambiental como factor determinante de la salud“, Revista Española de la Salud Pública, pp. 117127.

XVIII Ayuntamiento de Mexicali-IMIP (2006), Programa de Desarrollo Urbano de Centro de Población Mexicali, B.C., 2025, versión abreviada para consulta, Mexicali, Baja California. 